\title{
Two-Dimensional Binary Halftoned Optical Intensity Channels
}

\author{
Mohamed D.A. Mohamed and Steve Hranilovic \\ Dept. Electrical \& Computer Engineering \\ McMaster University \\ Hamilton, Ontario, Canada L8S 4K1 \\ Email: mdamohamed@grads.ece.mcmaster.ca, hranilovic@mcmaster.ca \\ appeared in IET Communications, \\ Special Issue on Optical Wireless Communication Systems, \\ Volume 2, Issue 1, pp. 11-17, January 2008.
}

\begin{abstract}
This paper considers the capacity of two-dimensional optical intensity channels in which transmit images are constrained to be binary-level. Examples of such links exist in holographic storage, page-oriented memories, optical interconnects, two-dimensional barcodes, as well as MIMO wireless optical links. Data are transmitted by sending a series of time-varying binary-level optical intensity images from transmitter to receiver. Strict spatial alignment between transmitter and receiver is not required nor is independence among the spatial channels assumed. Our approach combines spatial discrete multitone modulation developed for spatially frequency selective channels with halftoning to produce a binary-level output image. Data is modulated in spatial frequency domain as dictated by a water pouring spectrum over the optical transfer function as well as channel and quantization noise. A binary-level output image is produced by exploiting the excess spatial bandwidth available at the transmitter to shape quantization noise out of band. We present a general mathematical framework in which such systems can be analyzed and designed. In a pixelated wireless optical channel application, halftoning achieves $99.8 \%$ of the capacity of an equivalent unconstrained continuous amplitude channel using 1 megapixel arrays.
\end{abstract}

Index Terms: two-dimensional wireless optical channels, wireless infrared communications, optical intensity modulation, halftoning, holographic storage, page-oriented memories, optical interconnects, two-dimensional barcodes, MIMO wireless optical links, pixelated optical channels.

*This paper is a postprint of a paper submitted to and accepted for publication in IET Communications and is subject to Institution of Engineering and Technology Copyright. The copy of record is available at IET Digital Library. 


\section{Introduction}

Information is transmitted through optical intensity channels by modulating the instantaneous intensity of an optical source. For two-dimensional (2D) optical channels, the transmitted signal is a 2D intensity image and, as a result, must have non-negative amplitudes. Moreover, the average transmitted power is given by the average image amplitude rather than the average square amplitude as in conventional electrical channels. As a result of these amplitude constraints, the direct application of conventional signalling theory is seldom efficient.

Such 2D optical intensity channels have been used in a variety of applications such as holographic data storage [1-3], page-oriented optical recording [4,5], 2D barcodes [6, 7], and multiple-input/multiple-output (MIMO) wireless optical communications [8-11]. A major practical problem of many of these applications is the requirement of strict alignment between the transmitter and the receiver in order to avoid inter-channel interference $[1,2,12]$. Such a system is termed "pixel matched", i.e. each receive pixel images a single transmit pixel. Another practical limitation is the constraint on the average and peak optical power transmitted which is particularly important in applications where the optical signal can interfere with humans, as the case with MIMO wireless optical communications [8].

Recently, a signalling technique, which provides high spectral efficiencies without the need for strict spatial alignment, has been introduced for 2D intensity channels $[13,14]$. The only requirement is that the transmitter array is in the field-of-view of the receiver. Inter-channel interference is in fact, modelled and exploited at the transmitter and receiver through the use of spatio-temporal coding. The channel considered in $[13,14]$ is a class of MIMO wireless optical channels, and is termed pixelated wireless optical channel. In this previous work, the transmitter array is assumed to be able to generate multiple (e.g., 256) intensity levels. Additionally, the non-negativity constraint of the generated image is not considered explicitly, rather, amplitudes are clipped to ensure that the constraints are met.

In this paper, we extend the earlier work on pixelated wireless optical channel to the general case of $2 \mathrm{D}$ optical intensity channels by considering transmitter arrays, i.e., spatial 
light modulators (SLMs), in which each pixel is able to output a binary-level intensity, i.e., on or off. Such transmitters are far simpler than those previously assumed and can be operated at very high frame rates. The non-negativity constraint is treated explicitly through the design of modified halftoning algorithms. Thus, excess degrees of freedom in spatial frequency domain are exploited to provide binary-level output images which satisfy all amplitude constraints while maintaining the relaxation of the strict spatial alignment.

In Sec. 2, a brief introduction on 2D optical intensity channels is presented along with a channel model. Binary-level signalling over these channels is discussed in Sec. 3 along with an analysis for quantization noise and capacity of such links. A numerical example based on a measured pixelated wireless optical channel is presented in Sec. 4. The paper concludes in Sec. 5 with some directions for future work.

\section{$2 \quad 2 \mathrm{D}$ Optical Intensity Channels}

\subsection{Channel Model}

The transmitter is an SLM consisting of a 2D array of pixels, while the receiver consists of a 2D array of photodiode pixels. The receiver samples the spatial distribution of the optical intensity wavefront incident on its surface. Examples of imaging-type receivers are arrays of photodiodes, charge coupled device (CCD) cameras, and CMOS imagers. Several such arrays have been constructed for free-space and indoor wireless optical communications $[15,16]$, as well for high-speed imaging applications with rates near $10 \mathrm{kframes} / \mathrm{s}$ for arrays of over $10^{5}$ pixels [17].

We assume that imaging optics are employed, and that the optical axes of the transmitter and the receiver are aligned such that the receive image $r\left(n_{1}, n_{2}\right)$ is an orthographic projection of the transmit image $x\left(n_{1}, n_{2}\right)$, where $\left(n_{1}, n_{2}\right)$ are continuous spatial coordinates. According to scalar diffraction theory, the impact of the imaging system as well as the shape of transmit and receive pixels can be well modelled as a spatial low pass filter. This low pass filter is characterized by its point-spread function (PSF) [18]. That is, the PSF is the optical impulse 
response of the system which is modelled as a linear space-invariant system. Therefore, the channel model can be written as

$$
r\left(n_{1}, n_{2}\right)=x\left(n_{1}, n_{2}\right) \otimes h\left(n_{1}, n_{2}\right)+z\left(n_{1}, n_{2}\right)
$$

where $h\left(n_{1}, n_{2}\right)$ is the channel PSF, $z\left(n_{1}, n_{2}\right)$ is the channel noise, and $\otimes$ is $2 \mathrm{D}$ linear convolution. The channel model in (1) is a generalization of a previous model used in holographic data storage [3] and 2D optical recording [4], where the channel was modelled as a finiteextent 2D linear filter that represents the optical blur, or the 2D inter-symbol interference. The same model was used in wireless optical communication applications as well [13,14].

Two channel amplitude constraints are imposed on $x\left(n_{1}, n_{2}\right)$ : (i) a non-negativity constraint, $x\left(n_{1}, n_{2}\right) \geq 0$, that stems from the fact that the transmitted signal is an optical intensity signal, and (ii) $\mathbb{E}\left\{x\left(n_{1}, n_{2}\right)\right\} \leq P$, where $P$ is the average optical power, and $\mathbb{E}$ is the expectation operator.

\subsection{Spatial Discrete Multitone Modulation}

Discrete multitone (DMT) is a modulation scheme developed for frequency selective channels. Spatial discrete multitone (SDMT) modulation is the generalization of DMT to 2D spatial frequency [14]. An appealing advantage to 2D intensity channels is that SDMT is insensitive to the receiver sampling phase, and hence, strict alignment between the transmitter and receiver is not necessary.

Let the transmit image be $N_{1} \times N_{2}$ pixels and let $X$ be the discrete Fourier transform (DFT) of the transmit image. The complex data to be transmitted are loaded in the complex

frequency bins $X\left(k_{1}, k_{2}\right)$. Hermitian symmetry $X\left(k_{1}, k_{2}\right)=X^{*}\left(N_{1}-k_{1}, N_{2}-k_{2}\right)$ must be satisfied in order to guarantee real transmit images. According to this model, the transmit images are periodic in space. The receive image is equal to the linear convolution of the transmit image and the channel PSF. By appending a cyclic extension $[14,19]$ around the image, whose size is at least half the channel memory, the periodicity assumption of the DFT 
is satisfied and equalization is a complex multiplication per spatial frequency bin. In this case, the channel model (1) can be simplified in discrete spatial frequency domain $\left(k_{1}, k_{2}\right)$ as

$$
R\left(k_{1}, k_{2}\right)=X\left(k_{1}, k_{2}\right) H\left(k_{1}, k_{2}\right)+Z\left(k_{1}, k_{2}\right)
$$

where $R\left(k_{1}, k_{2}\right), X\left(k_{1}, k_{2}\right), H\left(k_{1}, k_{2}\right)$, and $Z\left(k_{1}, k_{2}\right)$ are the DFTs of the receive image, transmit image, PSF, and channel additive Gaussian noise respectively. Thus, the channel can be considered as a number of parallel Gaussian channels in spatial frequency domain, and the aggregate capacity, in the absence of amplitude constraints, is maximized by a water pouring algorithm over the spatial frequency channels [14].

Due to the non-negativity amplitude constraint of the spatial intensity domain, pouring power to the spatial frequency channels is not straight forward, and does not necessarily maximize the capacity $[13,14]$. The complexity of this technique is increased since it requires an SLM capable of outputting a continuous range of intensities with a high dynamic range, as is noted in electrical DMT systems.

\section{Binary-Level 2D Optical Channels}

In an effort to reduce the complexity of the transmitter, we confine our consideration to SLMs with binary-level output. Binary-level signalling over the 2D optical wireless channel simplifies not only the SLM design but also ensures that the channel non-negativity constraint can be easily met since dark/bright pixels are only transmitted over the channel.

Two popular examples of such binary-level SLMs include digital micro-mirror devices (DMD) and arrays of vertical-cavity surface-emitting lasers (VCSELs). Commercial DMD can operate at switching speeds of nearly $10 \mathrm{kHz}$ with array sizes of $2048 \times 1152$ mirrors [20]. A 540-element array with 1080 VCSELs has been produced for optical interconnect in which each pixel can be modulated in excess of $200 \mathrm{Mbps}$ [21]. An integrated solid-state array of transceivers has been designed to operate at 155 Mbps per pixel, where arrays of resonant cavity light-emitting diodes are bonded to arrays of CMOS driver circuits [22]. 
The high frame rates permit many channel uses per second, while the large number of pixels gives many spatial degrees of freedom. Since the PSF of the channel is typically spatially low pass, it is not possible to transmit data in high spatial frequency modes as these modes are effectively filtered by the channel. The high-frequency modes are termed the outof-band region, while the low-frequency modes which carry independent data are termed the in-band region. In this section, we propose a method that utilizes these out-of-band spatial modes to satisfy the channel non-negativity constraint by producing binary-level output images while achieving high communication rates that approach the rates of unconstrained continuous SLM transmission.

\subsection{Digital Image Halftoning}

In image processing, digital image halftoning is defined as the process of converting a continuous-tone image to a binary-level one which is perceptually close to the original continuous image $[23,24]$. The perceptual quality of the image is dominated by the low spatial frequency region since the human visual system is more sensitive to this region of the spectrum. Therefore, the power spectral density (PSD) of the quantization noise is shaped to the high spatial frequency region where the human visual system is not sensitive.

Error diffusion halftoning is the most popular halftoning algorithm first proposed empirically by Floyd and Steinberg in 1975 [25]. Later, Anastassiou defined a rigorous unifying framework linking $\Delta \Sigma$ modulation and error diffusion halftoning [26]. He showed that the error diffusion algorithm is the extension of $\Delta \Sigma$ modulation to two dimensions and that both algorithms are oversampled analog-to-digital converters that rely on the spectral shaping of quantization noise. The error diffusion system used in this work is surrounded by a dashed box in Fig. 1. The error diffusion feedback filter $j\left(n_{1}, n_{2}\right)$ was chosen empirically by Floyd, however, others have presented more rigorous designs based on classical filter theory [27]. An all-optical implementation of the error diffusion algorithm has been described in [28], where all pixel quantization decisions are computed in parallel.

In $2 \mathrm{D}$ optical intensity channels, the perceptual quality of the transmitted images is not 


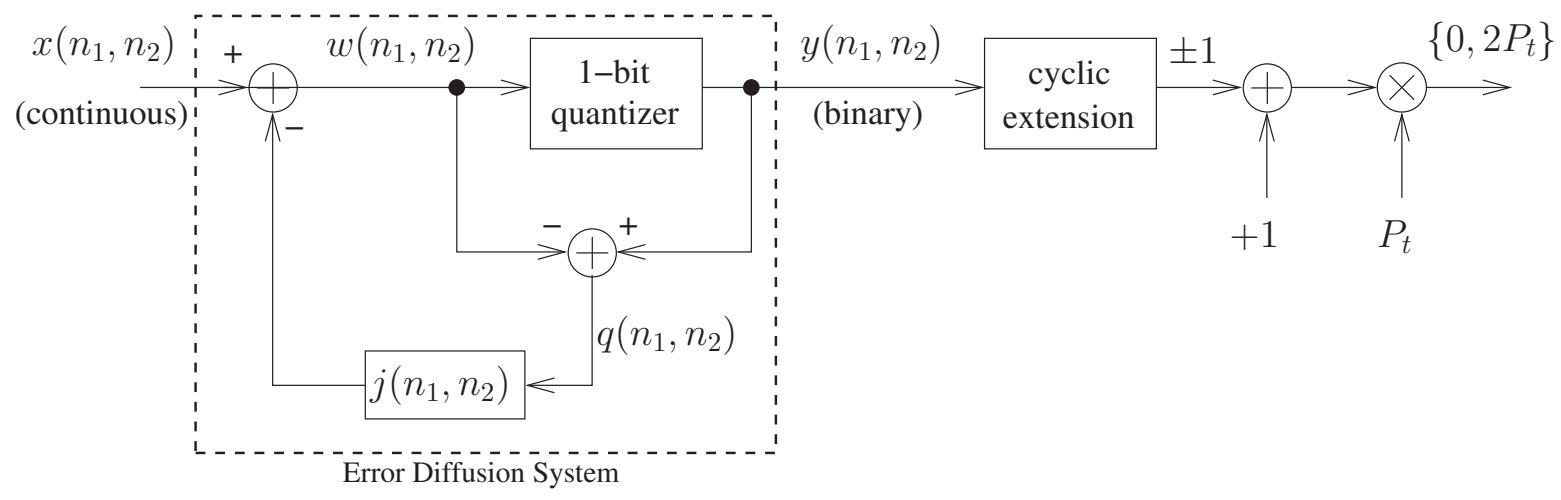

Figure 1: Halftoned SDMT over 2D optical intensity channels.

of concern. Instead, the goal is to design a series of images which maximize the rate at which reliable communication can take place over the channel. Since the channel PSF is analogous to the low-pass human visual system, data are modulated into spatial frequency bins where the channel attenuation is low. Halftoning is then used to produce a binary-level transmit image in which the quantization noise is shaped to the out-of-band frequency bins that are heavily attenuated by the channel PSF. This high frequency quantization noise will be filtered out by the channel and the received image will be a continuous-tone image which carries the transmitted data along with some residual quantization noise.

In Fig. 1, let $q$ be the quantizer error, and $\tilde{q}=y-x$ be the closed loop quantization noise, where $x$ and $y$ are the input continuous-tone image and the output halftoned image respectively. The analysis of this error diffusion system is not straightforward because the binary-level quantizer is a highly nonlinear element. A conventional assumption is to linearize the quantizer by assuming that the quantizer error $q$ is white with variance $\sigma_{q}^{2}$ and signal independent [29], [30, Chapter 14]. Once linearized, the following PSD of the quantization noise $\tilde{q}$ can be easily deduced

$$
\begin{aligned}
\Phi_{\tilde{q}}\left(k_{1}, k_{2}\right) & =\Phi_{q}\left(k_{1}, k_{2}\right)\left|1-J\left(k_{1}, k_{2}\right)\right|^{2} \\
& =N_{1} N_{2} \sigma_{q}^{2}\left|1-J\left(k_{1}, k_{2}\right)\right|^{2}
\end{aligned}
$$

where $\Phi_{q}\left(k_{1}, k_{2}\right)=N_{1} N_{2} \sigma_{q}^{2}$ is the PSD of the quantizer error, and $J\left(k_{1}, k_{2}\right)$ is the DFT of 
the feedback filter. That is, the power of the quantizer error is shaped by the noise power shaping function $\left|1-J\left(k_{1}, k_{2}\right)\right|^{2}$.

In order to shape the quantizer error power to the out-of-band region, the error diffusion feedback filter $J\left(k_{1}, k_{2}\right)$ is chosen to be a unity DC-gain filter such that the noise power shaping function $\left|1-J\left(k_{1}, k_{2}\right)\right|^{2}$ will have a null at DC. The total quantization noise power, in all complex frequency bins, after noise shaping is given by:

$$
\begin{aligned}
\sum_{k_{1}, k_{2}} \Phi_{\tilde{q}} & =N_{1} N_{2} \sigma_{q}^{2} \sum_{k_{1}, k_{2}}\left[1-J\left(k_{1}, k_{2}\right)\right]^{*}\left[1-J\left(k_{1}, k_{2}\right)\right] \\
& =\left(N_{1} N_{2}\right)^{2} \sigma_{q}^{2}\left(1+\mathcal{E}_{j}-2 j(0,0)\right)
\end{aligned}
$$

where

$$
\mathcal{E}_{j}=\sum_{n_{1}, n_{2}}\left|j\left(n_{1}, n_{2}\right)\right|^{2}
$$

is the feedback filter energy, and the fact that $\sum J\left(k_{1}, k_{2}\right)=N_{1} N_{2} j(0,0)$ is used.

Therefore, the total quantization noise power increases with the filter energy $\mathcal{E}_{j}$. However, high values of $\mathcal{E}_{j}$ are not problematic as long as the noise is well shaped to the out-of-band region that is attenuated by the channel PSF. The key point is to maximize the channel capacity, defined in Sec. 3.2, and not to minimize the total quantization noise power.

\subsection{Halftoned Spatial Discrete Multitone Modulation}

The binary-level SDMT communication system based on halftoning is shown in Fig. 1. The SDMT transmit image is formed as described in Sec. 2.2 and is the input of the error diffusion system. A cyclic extension is added to the halftoned image and the frame is biased by a constant bias of +1 to satisfy the non-negativity constraint. Finally the entire frame is multiplied by $P_{t}$ to scale the output levels to $\left\{0,2 P_{t}\right\}$. Since the input continuous SDMT image $x\left(n_{1}, n_{2}\right)$ is assumed to be zero mean, the output halftoned binary image $y\left(n_{1}, n_{2}\right)$ is also zero mean, and hence the average transmit optical power is equal to $P_{t}$. 
The received image, as given by (2), can be rewritten as

$$
R\left(k_{1}, k_{2}\right)=P_{t}\left[X\left(k_{1}, k_{2}\right)+\tilde{Q}\left(k_{1}, k_{2}\right)+N_{1} N_{2} \delta\left(k_{1}, k_{2}\right)\right] H\left(k_{1}, k_{2}\right)+Z\left(k_{1}, k_{2}\right)
$$

Dividing (5) by $P_{t} H\left(k_{1}, k_{2}\right)$ yields,

$$
\frac{R\left(k_{1}, k_{2}\right)}{P_{t} H\left(k_{1}, k_{2}\right)}=\left[X\left(k_{1}, k_{2}\right)+N_{1} N_{2} \delta\left(k_{1}, k_{2}\right)\right]+\tilde{Q}\left(k_{1}, k_{2}\right)+\tilde{Z}\left(k_{1}, k_{2}\right)
$$

where

$$
\tilde{Z}\left(k_{1}, k_{2}\right)=\frac{Z\left(k_{1}, k_{2}\right)}{P_{t} H\left(k_{1}, k_{2}\right)}
$$

is the effective channel Gaussian noise.

That is, the effective received image is equal to a DC-biased version of the transmit image contaminated by two noise components: $\tilde{Q}\left(k_{1}, k_{2}\right)$ and $\tilde{Z}\left(k_{1}, k_{2}\right)$ whose PSDs are $\Phi_{\tilde{q}}\left(k_{1}, k_{2}\right)$ and $\Phi_{\tilde{z}}\left(k_{1}, k_{2}\right)$ respectively. By further assuming that the quantizer error is Gaussian, then $\Phi_{\tilde{q}}\left(k_{1}, k_{2}\right)+\Phi_{\tilde{z}}\left(k_{1}, k_{2}\right)$ defines the Gaussian noise "bowl" over which the total available electrical power is poured in order to maximize the aggregate channel capacity. If $\Phi_{\tilde{q}}\left(k_{1}, k_{2}\right)>>\Phi_{\tilde{z}}\left(k_{1}, k_{2}\right)$ in the in-band region, the quantization noise dominates and the system is termed quantization noise-limited. On the other hand, if $\Phi_{\tilde{q}}\left(k_{1}, k_{2}\right)<<\Phi_{\tilde{z}}\left(k_{1}, k_{2}\right)$ in the in-band region, the channel noise dominates and the system is then termed optical power-limited.

Notice that the non-negativity constraint is no longer an issue while pouring power to the bowl $\Phi_{\tilde{q}}\left(k_{1}, k_{2}\right)+\Phi_{\tilde{z}}\left(k_{1}, k_{2}\right)$, as was the case in $[13,14]$, because the output image is a binary one. Let $\Phi_{x}\left(k_{1}, k_{2}\right)$ be the electrical power allocated to the complex frequency bin $\left(k_{1}, k_{2}\right)$ such that the total frame electrical power is equal to $\frac{1}{N_{1} N_{2}} \sum \Phi_{x}\left(k_{1}, k_{2}\right)=\sigma_{x}^{2} N_{1} N_{2}$ for some fixed input electrical power $\sigma_{x}^{2}$. Then, the aggregate system capacity $C$ is given by

$$
C=\frac{1}{2} \sum_{k_{1}=0}^{N_{1}-1} \sum_{k_{2}=0}^{N_{2}-1} \log \left(1+\frac{\Phi_{x}\left(k_{1}, k_{2}\right)}{\Phi_{\tilde{q}}\left(k_{1}, k_{2}\right)+\Phi_{\tilde{z}}\left(k_{1}, k_{2}\right)}\right)
$$

where $\Phi_{x}\left(k_{1}, k_{2}\right)$ is calculated by the water pouring algorithm to maximize the capacity $C$ 
[31], and no power is poured to the DC-bin, i.e. $\Phi_{x}(0,0)=0$, because a constant DC-level of $P_{t}$ is added to each frame.

Notice that the expression in (6) for the capacity is only valid under the aforementioned assumptions of signal-independent, white, Gaussian quantizer error. The larger the total input signal electrical power, the more likely this assumption is to fail. In Sec. 3.3, we deduce an upper bound on the total input electrical power and feedback filter energy that are necessary to ensure the consistency of the system model.

\subsection{Linearized Analytical Model}

Using the signal-independence and whiteness assumptions of the quantizer error, the variance $\sigma_{w}^{2}$ of the input to the quantizer can be written as

$$
\sigma_{w}^{2}=\sigma_{x}^{2}+\sigma_{q}^{2} \mathcal{E}_{j}
$$

where $\sigma_{x}^{2}$ is the average electrical power of the input SDMT signal as shown in Fig. 1.

The availability of some information about the statistical properties of the input SDMT signal can further aid the estimation of the quantizer error power $\sigma_{q}^{2}$. The amplitude of DMT signals is known to closely approximate Gaussian distributed noise [32, Sec. 3.3]. Therefore, from the Gaussianity assumption of the quantizer error, the input $w$ to the quantizer is Gaussian distributed as well, and the quantizer error power is given by

$$
\begin{aligned}
\sigma_{q}^{2} & =\int_{-\infty}^{0} f_{w}(w)(-1-w)^{2} d w+\int_{0}^{\infty} f_{w}(w)(1-w)^{2} d w \\
& =1+\sigma_{w}^{2}-4 \sigma_{w} / \sqrt{2 \pi}
\end{aligned}
$$

where $f_{w}(w)$ is a zero-mean Gaussian probability density function with variance $\sigma_{w}^{2}$. Solving (7) and (8) for $\sigma_{w}$,

$$
\sigma_{w}=\frac{-\sqrt{2 / \pi}+\sqrt{2 / \pi+\left(1 / \mathcal{E}_{j}-1\right)\left(1+\sigma_{x}^{2} / \mathcal{E}_{j}\right)}}{1 / \mathcal{E}_{j}-1}
$$


Therefore, $\sigma_{q}^{2}$ can be estimated from knowledge of $\sigma_{x}^{2}$ by using (8) and (9). For $\sigma_{w}$ to be real, the discriminant under the square root in (9) must be positive, and hence, the following upper bound on $\sigma_{x}^{2}$ exists when $\mathcal{E}_{j}>1$,

$$
\sigma_{x}^{2} \leq \mathcal{E}_{j}\left[\frac{2}{\pi\left(1-1 / \mathcal{E}_{j}\right)}-1\right]
$$

Since $\sigma_{x}^{2} \geq 0$, then so too is the right hand side of (10). Hence, we have the following upper bound on the feedback filter energy $\mathcal{E}_{j}$

$$
\mathcal{E}_{j} \leq \frac{\pi}{\pi-2} \approx 2.75
$$

That is, for our model to be consistent with the assumptions made, the feedback filter energy cannot exceed 2.75, and the input electrical power cannot exceed the bound in (10) when $\mathcal{E}_{j}>1$.

\section{Example: Pixelated Wireless Optical Channel}

In this section, a specific example of a 2D optical communication channel, namely, the pixelated wireless optical channel, is studied. The pixelated wireless optical channel is introduced in $[13,14]$, where each transmitter element is assumed to be able to generate 256 intensity levels. Additionally, the non-negativity constraint of the generated image was not considered explicitly, rather, amplitudes are clipped to ensure non-negativity. In this section, halftoned SDMT is applied to the channel measured in $[13,14]$, and the obtained channel capacity is compared to the results of $[13,14]$. In Sec. 4.1, the channel parameters are defined. The error diffusion feedback filter is designed in Sec. 4.2, and numerical results and discussion are presented in Sec. 4.3. 


\subsection{Simulation Setup}

In Sec. 3.1, it was assumed that the quantizer error is white and signal independent with variance $\sigma_{q}^{2}$. Similarly, it is assumed in our simulations that the channel noise is white, Gaussian, and signal independent with variance $\sigma_{z}^{2}$. Therefore the PSD of the effective noise $\tilde{Z}$ is equal to

$$
\Phi_{\tilde{z}}\left(k_{1}, k_{2}\right)=\frac{\sigma_{z}^{2} N_{1} N_{2}}{\left|P_{t} H_{0} H\left(k_{1}, k_{2}\right)\right|^{2}}=\frac{N_{1} N_{2}}{\left|\rho H\left(k_{1}, k_{2}\right)\right|^{2}}
$$

where $H_{0}$ is the DC-gain of the unity DC-gain PSF, $H\left(k_{1}, k_{2}\right)$, and

$$
\rho=P_{t} H_{0} / \sigma_{z}
$$

Substituting from (3) and (12) in (6), the capacity is equal to

$$
C=\frac{1}{2} \sum_{k_{1}=0}^{N_{1}-1} \sum_{k_{2}=0}^{N_{2}-1} \log \left(1+\frac{\Phi_{x}\left(k_{1}, k_{2}\right)}{N_{1} N_{2} \sigma_{q}^{2}\left|1-J\left(k_{1}, k_{2}\right)\right|^{2}+N_{1} N_{2} /\left|\rho H\left(k_{1}, k_{2}\right)\right|^{2}}\right)
$$

Notice that the system is quantization noise-limited if $\rho>>1 / \sigma_{q}$, and is optical powerlimited if $\rho<<1 / \sigma_{q}$.

In our simulations, we have assumed that both the transmitter and receiver have the same number of pixels and that spatial synchronization is achieved through appropriate scaling of the imaging optics [14]. Therefore, the transmitter and receiver have the same spatial Nyquist region. The transmitted image is required to be in the field-of-view of the receiver, while strict alignment between the transmitter and receiver is not necessary. The simulations are done for a square frame $N_{1}=N_{2}=N$, and a unity DC-gain Gaussian PSF,

$$
H\left(k_{1}, k_{2}\right)=\exp \left(-\frac{k_{1}^{2}}{2 w_{h_{1}}^{2}}-\frac{k_{2}^{2}}{2 w_{h_{2}}^{2}}\right)
$$

where $w_{h_{1}}$ and $w_{h_{2}}$ are measures of the PSF widths. In all simulations, the channel parameters are taken from a measured pixelated wireless optical channel [14], and the parameters are presented in Table 1. 


\begin{tabular}{cccc}
\hline$N$ & $w_{h_{1}}$ & $w_{h_{2}}$ & $\rho$ \\
\hline 154 & 41.01 & 41.85 & 56.05 \\
\hline
\end{tabular}

Table 1: Measured parameters of a pixelated wireless optical channel in $[13,14]$. The measured $\rho$ is a worst case, calculated at the highest noise variance measured in $[13,14]$.

All the measurements presented in Table 1 were done for a single receiver array with $N=154$ pixels. For a given imager size, increasing the value of $N$ by a factor $\alpha$ implies a corresponding decrease in the pixel area by a factor $\alpha^{2}$. This in turn results in (i) decreasing the DC-gain of the PSF, i.e. $H_{0}$, by $\alpha^{2}$, and (ii) decreasing the channel noise power, i.e. $\sigma_{z}^{2}$, by $\alpha^{2}$. This can be attributed to the fact that both the average optical power collected by the pixel and the pixel noise variance are proportional to the pixel area [33,34]. Notice that the PSF in (15) is the product of the transmitter pixel shape, the imaging optics transfer function, and the receiver pixel shape [14]. In light of (13), $\rho$ decreases by a factor of $\alpha$, and the following appropriate scaling of $\rho$ is used during simulations

$$
\rho_{N}=\frac{\rho_{154}}{\alpha}=\rho_{154} \times \frac{154}{N}
$$

where $\rho_{N}$ is the value of $\rho$ when the imager is $N \times N$ pixels, and $\rho_{154}$ is the value of $\rho$ when the imager is $154 \times 154$ pixels, given in Table 1 . Notice that the effect of changing the pixel area on the bandwidth of the PSF is negligible due to the very small pixel size.

\subsection{Halftoning Design}

In this example, the following form for the error diffusion feedback filter is proposed

$$
j\left(n_{1}, n_{2}\right)=\left[\begin{array}{cc}
0 & a \\
a & 1-2 a
\end{array}\right] \longleftrightarrow J\left(k_{1}, k_{2}\right)
$$

This filter is symmetric, causal, has unity DC-gain, and is among the simplest feedback filters that can be used for digital halftoning. The short length of the filter makes it faster and less complex to implement in real time. Another attractive feature is that a single parameter, $a$, 
indexes the entire family of filters. In this case,

$$
\mathcal{E}_{j}=\sum\left|j\left(n_{1}, n_{2}\right)\right|^{2}=6 a^{2}-4 a+1
$$

Substituting from (18) in the bound (11), $a$ is restricted to the interval

$$
-0.3<a<0.968
$$

in order for our model to be consistent. Higher values of $|a|$ can saturate the binary-level quantizer and hence affects the stability of the error diffusion system in Fig. 1.

A cross-section for $k_{1}=k_{2}$ through the noise power shaping function $\left|1-J\left(k_{1}, k_{2}\right)\right|^{2}$ is plotted for different values of $a$ in Fig. 2. It is straight forward to show that for $a=1 / 3, \mathcal{E}_{j}$ is minimized. Consequently, $a=1 / 3$ minimizes the total quantization noise power, in (4). From the figure it is also clear that maximizing $a$ is desirable, within (19), in order to widen the low quantization noise region around DC. Recall that the goal is to minimize the residual quantization noise in the spatial frequency bins which carry data rather than minimizing the total quantization noise power. Thus, $a=1 / 3$ is not necessarily the best choice for $J$.

As the electrical power $\sigma_{x}^{2}$ increases, so too does the dynamic range of the input and the likelihood of quantizer saturation and system instability. When $a>2 / 3, \mathcal{E}_{j}>1$ and the bound in (10) is necessary. In the simulation results that follow, $\sigma_{x}^{2}=0.1$ is used which satisfies the upper bound (10) for all values of $a$ considered.

An example of a continuous-tone image is shown in Fig. 3 for $N=512$, along with the corresponding halftoned image and their spectra. The complex data to be transmitted are loaded to the low frequency bins of the continuous-tone image as implied by the water pouring allocation. By comparing the spectra in Figs. 3(c) and 3(d), it is evident that the data can be recovered from the low frequency region of the halftoned binary-level image. A great majority of the quantization noise power is shaped to the out-of-band region, which is indicated by the four high power corners of the halftoned image spectrum. In this case, the system is optical power-limited as the effect of quantization noise is mild, and the shape of 


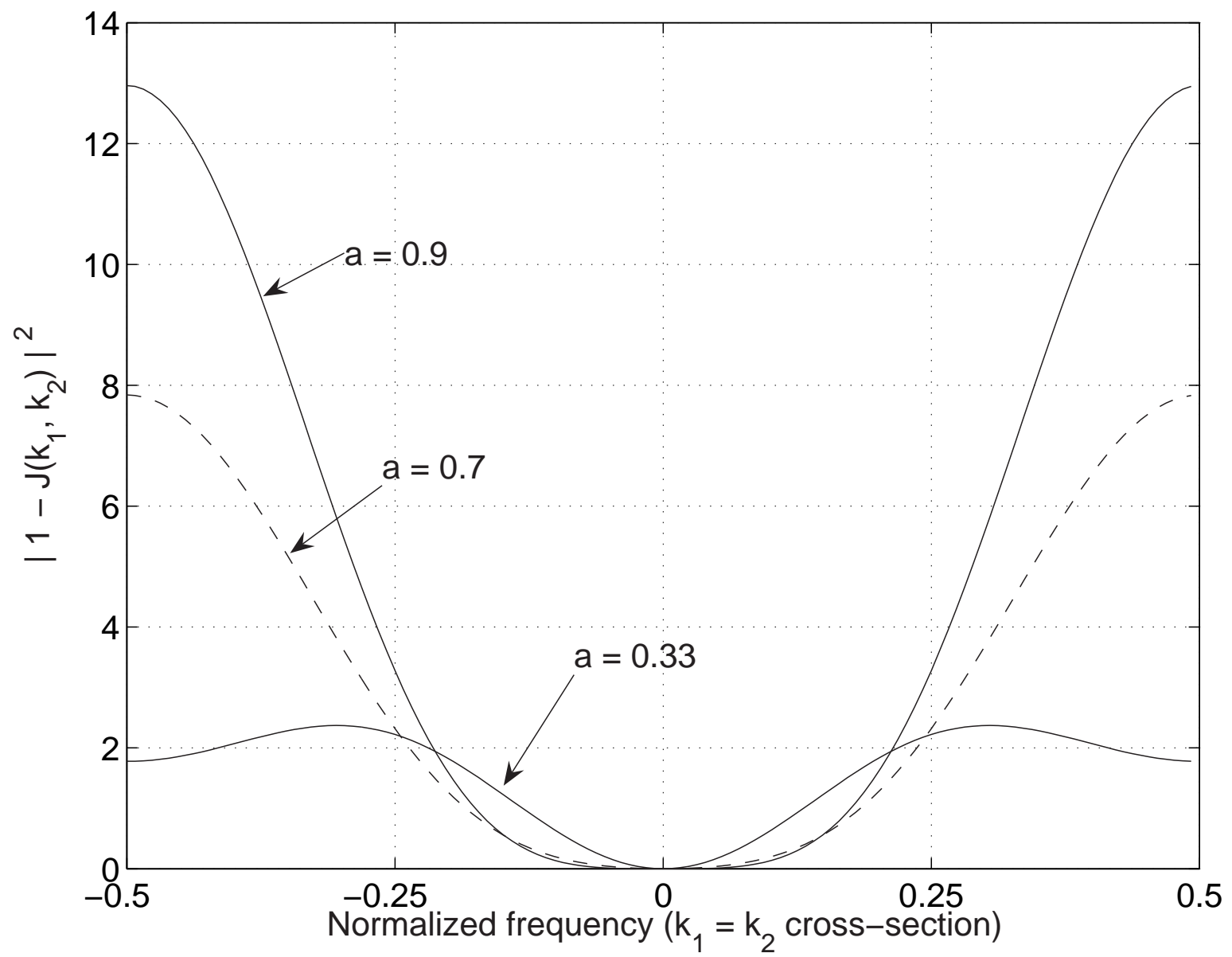

Figure 2: Cross-sections of the noise power shaping function, $\left|1-J\left(k_{1}, k_{2}\right)\right|^{2}$, for $k_{1}=k_{2}$ and a variety of feedback filter parameters $a$. 
the in-band power allocation is dominated by the PSF.

A similar example is shown in Fig. 4 for $N=256$. In this case, the system is quantization noise-limited due to the reduced number of out-of-band spatial modes to which quantization noise can be shaped. As a result, the shape of the in-band power allocation is dominated by the quantization noise shaping function $\left|1-J\left(k_{1}, k_{2}\right)\right|^{2}$. Qualitatively, data can not be fully recovered from the low frequency region of the spectrum of the halftoned image because of the in-band quantization noise. As will be shown in Sec. 4.3, the reduction in capacity resulting from reducing $N$ from 512 to 256 is nearly $51.7 \%$.

Notice that the capacity of the halftoned SDMT system, or any finite-level SDMT system, is upper bounded by that of a continuous SDMT transmitter with the same $\sigma_{x}^{2}$ neglecting the non-negativity amplitude constraints. This upper bound is given by (14) with $\sigma_{q}^{2}=0$.

\subsection{Discussion}

In this section, the capacity of the halftoned SDMT system is calculated for $\sigma_{x}^{2}=0.1$ and the system parameters given in Table 1 by two different procedures: (i) by using our linearized model to estimate $\sigma_{q}^{2}$, as in (8), and (ii) by simulations, where $\Phi_{\tilde{q}}\left(k_{1}, k_{2}\right)$ is estimated by averaging over 1000 randomly generated frames. Notice that the measured quantization noise $\operatorname{PSD}, \Phi_{\tilde{q}}\left(k_{1}, k_{2}\right)$, affects the water pouring bowl which in turn affects the power allocation over the complex frequency bins which again impacts the quantization noise. As a result, the iterative scenario shown in Fig. 5 is used during simulations to allow $\Phi_{\tilde{q}}\left(k_{1}, k_{2}\right)$ to converge to the correct value for power allocation.

The results obtained from our linearized analytical model are compared to simulations in Fig. 6. It is evident that both model and simulations are in close agreement. Notice the capacity increases with the number of pixels $N^{2}$. This is due to the fact that the size of the out-of-band region increases with $N$, and hence the quantization noise can be shaped further from the data bearing region as in Figs. 3 and 4. However, the capacity saturates to a limiting value as $N$ increases. This is due to the fact that, although the number of channels increases as $N^{2}$, the signal-to-noise ratio (proportional to $\rho^{2}$ ) decreases at the same 


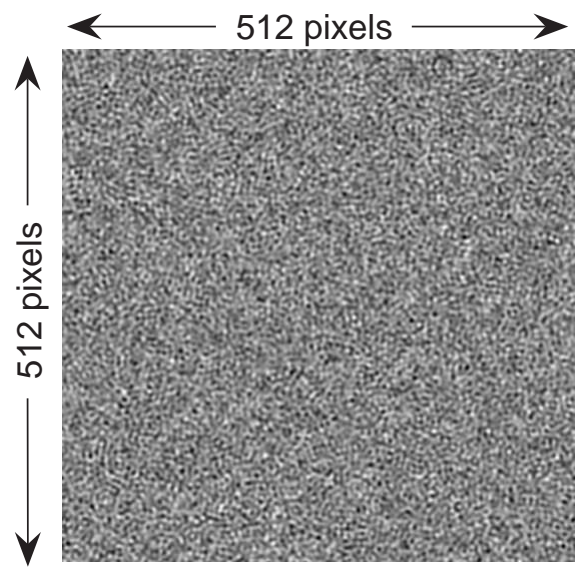

(a) continuous-tone image

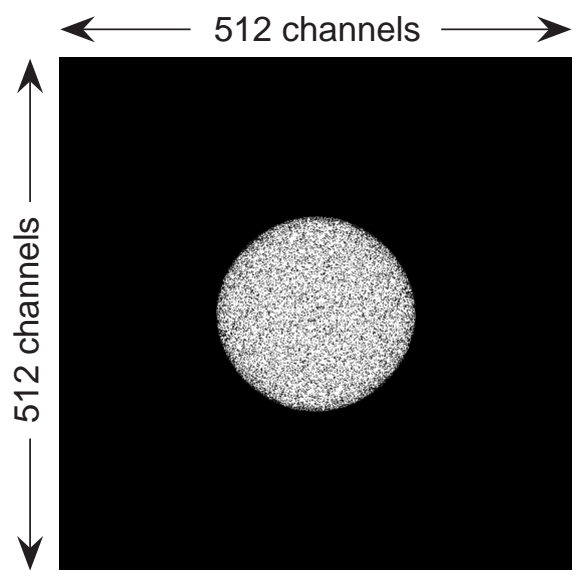

(c) continuous-tone image spectrum

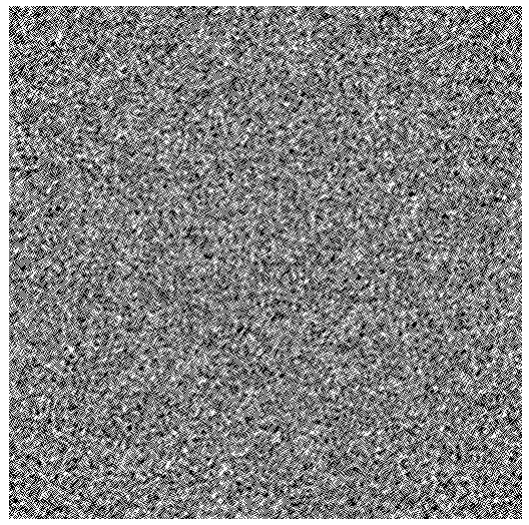

(b) halftoned binary-level image

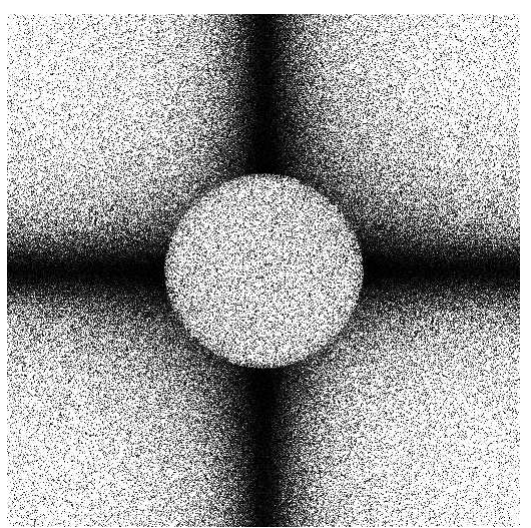

(d) halftoned binary-level image spectrum

Figure 3: An example continuous-tone image (a), corresponding halftoned image (b), and their spectra (c), (d) for $N=512, a=0.9, \sigma_{x}^{2}=0.1, w_{h_{1}}$ and $w_{h_{2}}$ as given in Table 1 , and $\rho$ as given by (16). This is an optical power-limited system. 


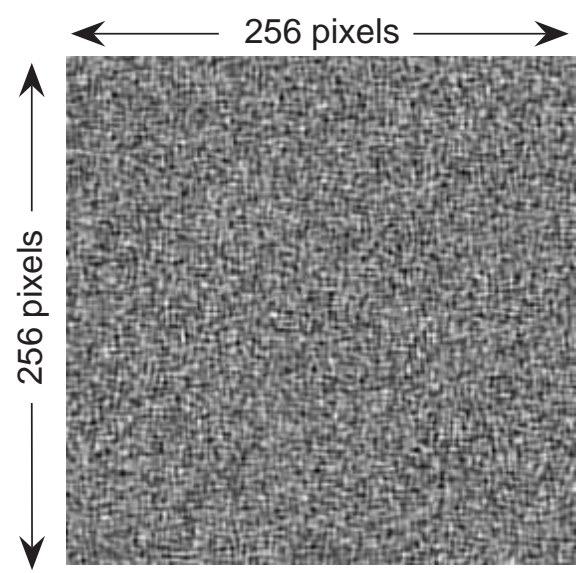

(a) continuous-tone image

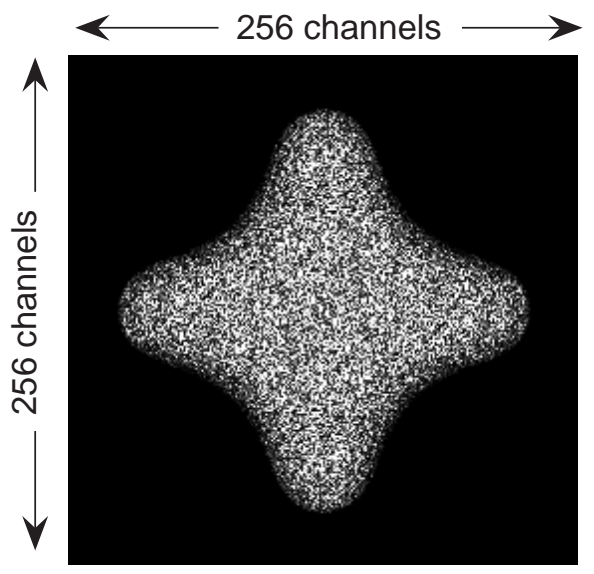

(c) continuous-tone image spectrum

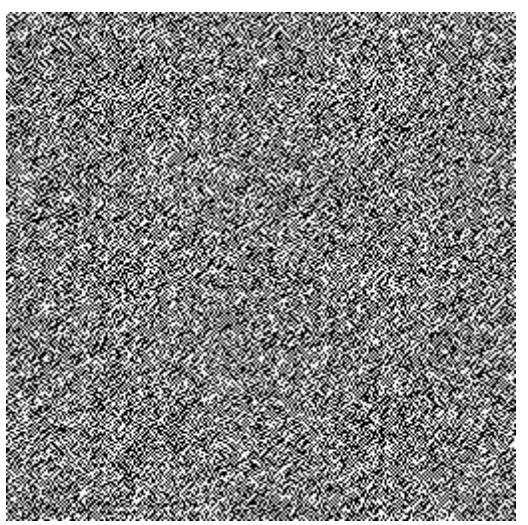

(b) halftoned binary-level image

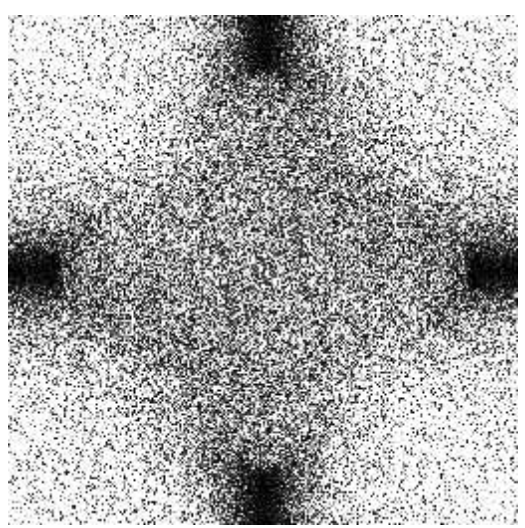

(d) halftoned binary-level image spectrum

Figure 4: An example continuous-tone image (a), corresponding halftoned image (b), and their spectra (c), (d) for $N=256, a=0.9, \sigma_{x}^{2}=0.1, w_{h_{1}}$ and $w_{h_{2}}$ as given in Table 1 , and $\rho$ as given by (16). This is a quantization noise-limited system. 


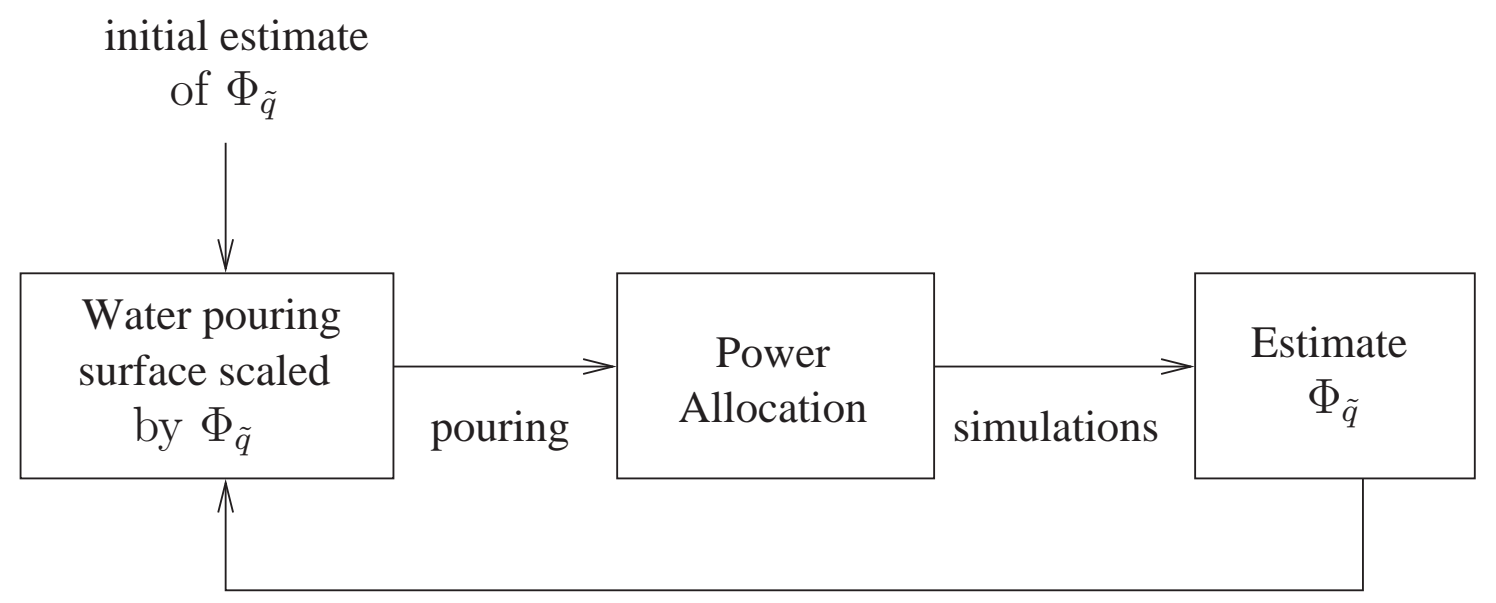

Figure 5: Iterations used in simulations to evaluate $\Phi_{\tilde{q}}\left(k_{1}, k_{2}\right)$ and the power allocation.

rate as in (16). Additionally, larger values of $a$ lead to high channel capacities. This is due to the fact seen in Fig. 2, that larger $a$ allows for lower residual quantization noise in the band of interest. Notice however, as implied by (10), as a increases, $\sigma_{x}^{2}$ must decrease to ensure stability of the halftoning modulator.

Fig. 6 compares the capacity of the halftoned SDMT system to an upper bound obtained by employing a continuous unconstrained SDMT transmitter with the same $\sigma_{x}^{2}$. The gap between the capacities of the binary-level transmission and the continuous-amplitude transmission diminishes as $N$ increases. This gap approaches zero in the limit $N \rightarrow \infty$ as the in-band quantization noise also approaches zero on this limit. Thus, for a given PSF, increasing the number of excess spatial degrees of freedom allows a binary-level SDMT transmitter to approach the same capacity as the optical power-limited regime.

Fig. 6 also compares the capacity of the halftoned SDMT system to that of the experimental 256-level SDMT system measured in [14] at $N=154$. The coded rate achieved in [14] is also indicated at the same value of $N$. The binary-level system with $a=0.9$ achieves $57.37 \%$ of the capacity of the 256-level system for $N=154$. Thus, for small $N$, quantization noise-limited channels benefit greatly from multi-level output. The capacity of the continuous system is an upper bound for both the 256-level and the binary systems. At higher values of $N$, the capacity of the binary system approaches the continuous-amplitude upper bound. For instance, the binary system $(a=0.9)$ achieves approximately $92.6 \%$ of 


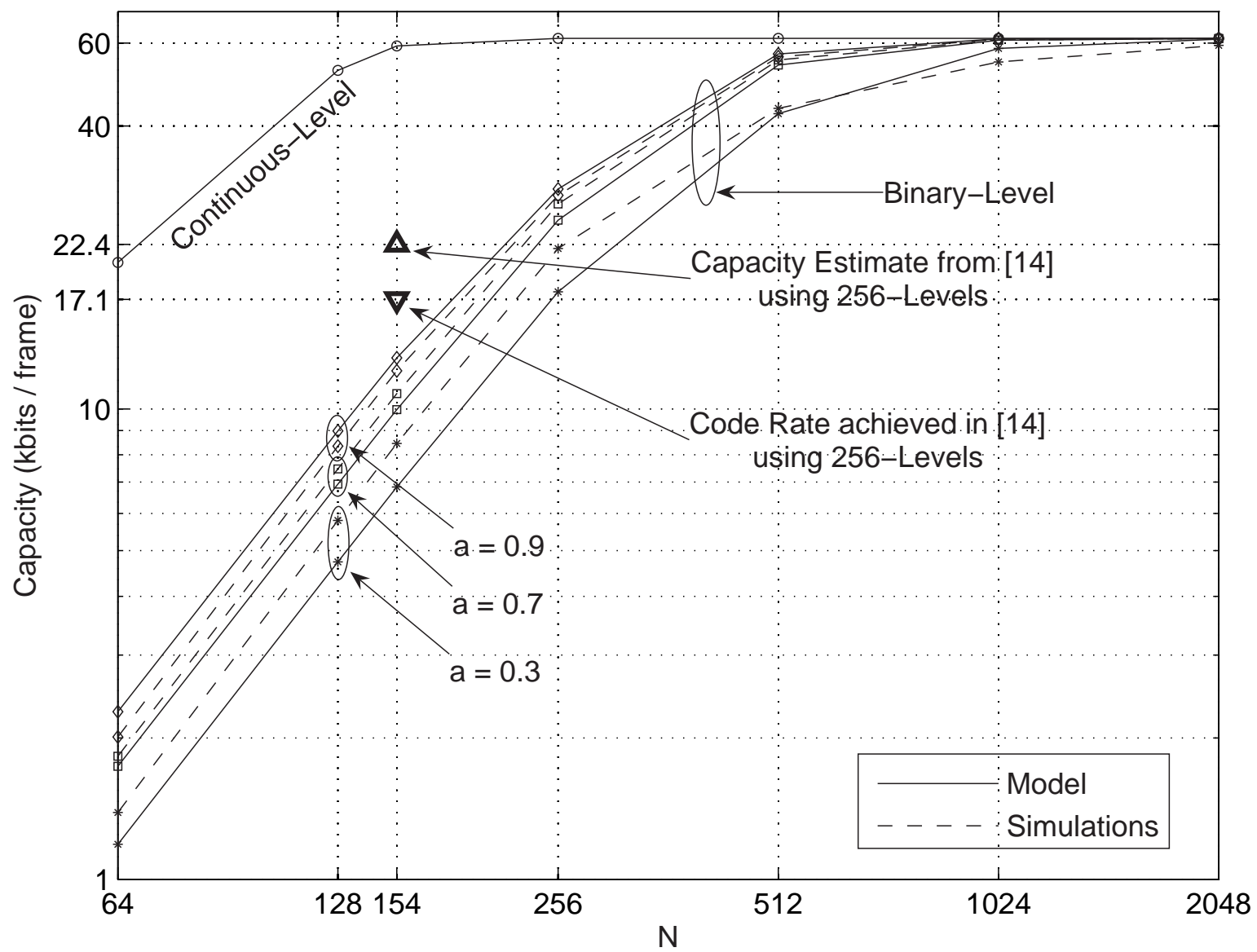

Figure 6: Capacity of the pixelated channel versus $N=N_{1}=N_{2}$ with $\sigma_{x}^{2}=0.1$. Results of the linearized analytical model in Sec. 3.3 are compared to those from simulations, and to those of a continuous SDMT transmitter without non-negativity constraint. The capacity estimate $(\triangle)$ and code rate $(\nabla)$ achieved in [14] using 256-level transmitter are also shown at $N=154$. 
the upper bound at $N=512$, and nearly $99.8 \%$ at $N=1024$. This suggests that employing more than two quantization levels is not necessary as $N$ gets large, because the system is optical power-limited in this case, and quantization noise is effectively shaped out-of-band.

\section{Conclusion}

The capacity and data rates of point-to-point $2 \mathrm{D}$ optical intensity channels can be significantly improved by using spatial degrees of freedom. In this work, we have considered the design of these channels in which only binary-level optical intensity transmitters are used. The approach proposed combines spatial discrete multitone modulation with halftoning ideas from image processing. Using a simple error diffusion filter, we demonstrate that the capacity of the channel using a binary-level transmitter approaches that when using a fictitious continuous transmitter that is capable of transmitting positive and negative amplitudes. In practice, the capacities reported here could be approached using spatio-temporal coding of the data bins, as was done in [14] for a pixelated wireless optical channel. However, our goal in this work was to demonstrate that the fundamental limits of pixelated channels with binary-level transmission are significant and merit continued study.

Currently, a prototype binary-level pixelated channel is being constructed by the authors using a DMD SLM with $1024 \times 768$ elements [20] and a mega-pixel CMOS imager. Additionally, reduced complexity coding and decoding techniques for these channels are also being explored to permit real-time operation. Potential applications of such intensity channels include holographic storage, page-oriented memories, optical interconnects, 2D barcodes, and MIMO wireless optical links. 


\section{References}

[1] R. M. Shelby, J. A. Hoffnagle, G. W. Burr, C. M. Jefferson, M.-P. Bernal, H. Coufal, R. K. Grygier, H. Gunther, R. M. Macfarlane, and G. T. Sincerbox, "Pixel-matched holographic data storage with megabit pages," Optics Letters, vol. 22, no. 19, pp. 1509 - 1511, Oct. 1997.

[2] G. W. Burr, J. Ashley, H. Coufal, R. K. Grygier, J. A. Hoffnagle, C. M. Jefferson, and B. Marcus, "Modulation coding for pixel-matched holographic data storage," Optics Letters, vol. 22, no. 9, pp. $639-641$, May 1997.

[3] S. Nabavi and B. V. K. V. Kumar, "Detection methods for holographic data storage," in Proceedings of the IEEE Optical Data Storage Topical Meeting, Montreal, QC, Canada, 23-26 Apr. 2006, pp. $156-158$.

[4] P. H. Siegel, "Information-theoretic limits of two-dimensional optical recording channels," in Proceedings of the IEEE Optical Data Storage Topical Meeting, Montreal, QC, Canada, 23-26 Apr. 2006, pp. 165- 167.

[5] L. Huang, G. Mathew, and T. C. Chong, "Channel modeling and target design for twodimensional optical storage systems," IEEE Transactions on Magnetics, vol. 41, no. 8, pp. 2414 - 2424, Aug. 2005.

[6] R. Villn, S. Voloshynovskiy, O. Koval, and T. Pun, "Multilevel 2-D bar codes: Toward highcapacity storage modules for multimedia security and management," to appear in the IEEE Transactions on Information Forensics and Security, 2006.

[7] W. Claycomb and D. Shin, "Using a two dimensional colorized barcode solution for authentication in pervasive computing," in Proceedings of the ACS/IEEE International Conference on Pervasive Services, Lyon, France, 26-29 June 2006, pp. 173 - 180.

[8] J. M. Kahn and J. R. Barry, "Wireless infrared communications," Proceedings of the IEEE, vol. 85, no. 2, pp. 263-298, Feb. 1997.

[9] D. C. O'Brien, S. Quasem, S. Zikic, and G. E. Faulkner, "Multiple input multiple output systems for optical wireless: Challenges and possibilities," Proceedings of SPIE, vol. 6304, 2006.

[10] J. Faucher, M. B. Venditti, and D. V. Plant, "Application of parallel forward-error correction in two-dimensional optical-data links," IEEE/OSA Journal of Lightwave Technology, vol. 21, no. 2, pp. 466 - 475, Feb. 2003.

[11] D. V. Plant and A. G. Kirk, "Optical interconnects at the chip and board level: challenges and solutions," Proceedings of the IEEE, vol. 88, no. 6, pp. $806-818,2000$.

[12] E. Bisaillon, D. F. Brosseau, T. Yamamoto, M. Mony, E. Bernier, D. Goodwill, D. V. Plant, and A. G. Kirk, "Free-space optical link with spatial redundancy for misalignment tolerance," IEEE Photonics Technology Letters, vol. 14, no. 2, pp. 242 - 244, 2002.

[13] S. Hranilovic and F. R. Kschischang, "Short-range wireless optical communication using pixelated transmitters and imaging receivers," in Proceedings of the IEEE International Conference on Communications, vol. 2, Paris, France, 20-24 June 2004, pp. 891-895.

[14] _ - "A pixelated MIMO wireless optical communication system," IEEE Journal of Selected Topics in Quantum Electronics, vol. 12, no. 4, pp. 859-874, Jul/Aug 2006. 
[15] B. S. Leibowitz, B. E. Boser, and K. S. J. Pister, "CMOS smart pixel for free-space optical communication," Proceedings of SPIE, vol. 4306A, pp. 21 - 26, Jan. 2001.

[16] D. C. O'Brien, G. E. Gaulkner, K. Jim, E. B. Zyamba, D. J. Edwards, M. Whitehead, P. Stavrinou, G. Parry, J. Bellon, M. J. Sibley, V. A. Lalithambika, V. M. Joyner, R. J. Samsudin, D. M. Holburn, and R. J. Mears, "High-speed integrated transceivers for optical wireless," IEEE Communications Magazine, vol. 41, no. 3, pp. 58 - 62, 2003.

[17] S. Kleinfelder, S. Lim, X. Liu, and A. E. Gamal, "A 10kframe/s 0.18 $\mu \mathrm{m}$ CMOS digital pixel sensor with pixel-level memory," in Proceedings of the IEEE International Solid-State Circuits Conference, San Francisco, CA, USA, 4-8 Feb. 2001, pp. 88 - 89.

[18] J. Goodman, Introduction to Fourier Optics, 1st ed. McGraw-Hill, June 1968.

[19] A. Peled and A. Ruiz, "Frequency domain data transmission using reduced computational complexity algorithms," in Proceedings of the IEEE International Conference on Acoustics, Speech, and Signal Processing, vol. 5, Denver, CO, USA, 9-11 Apr. 1980, pp. $964-967$.

[20] Texas Instruments, Digital Light Processing. http://www.dlp.com.

[21] M. B. Venditti, E. Laprise, J. Faucher, P.-O. Laprise, J. E. A. Lugo, and D. V. Plant, "Design and test of an optoelectronic-VLSI chip with 540-element receiver-transmitter arrays using differential optical signaling," IEEE Journal of Selected Topics in Quantum Electronics, vol. 9, no. 2, pp. 361 - 379, Mar./Apr. 2003.

[22] D. C. OBrien, G. E. Faulkner, E. B. Zyambo, K. Jim, D. J. Edwards, P. Stavrinou, G. Parry, J. Bellon, M. J. Sibley, V. A. Lalithambika, V. M. Joyner, R. J. Samsudin, D. M. Holburn, and R. J. Mears, "Integrated transceivers for optical wireless communications," IEEE Journal of Selected Topics in Quantum Electronics, vol. 11, no. 1, pp. 173 - 183, Jan./Feb. 2005.

[23] R. Ulichney, Digital Halftoning. Cambridge, MA, USA: MIT Press, 1987.

[24] B. L. Shoop, Photonic Analog-to-Digital Conversion. Berlin, Germany: Springer-Verlag, 2001.

[25] R. W. Floyd and L. Steinberg, "An adaptive algorithm for spatial gray scale," in SID Symposium Digest, Society for Information Display, 1975, pp. 36-37.

[26] D. Anastassiou, "Error diffusion coding for A/D conversion," IEEE Transactions on Circuits and Systems, vol. 36, no. 9, pp. 1175-1186, Sept. 1989.

[27] B. L. Shoop and E. K. Ressler, "An error diffusion neural network for digital image halftoning," in Proceedings of the IEEE Workshop on Neural Networks for Signal Processing, 31 Aug.-2 Sept. 1995, pp. 427-436.

[28] — - "Optimal error diffusion for digital halftoning using an optical neural network," in Proceedings of the IEEE International Conference on Image Processing, vol. 2, Austin, TX, USA, 13-16 Nov. 1994, pp. 1036-1040.

[29] S. R. Norsworthy, R. Schreier, and G. C. Temes, Eds., Delta-Sigma Data Converters: Theory, Design, and Simulation. NY, USA: Wiley-IEEE Press, Oct. 1996.

[30] D. A. Johns and K. W. Martin, Analog Integrated Circuit Design, 1st ed. John Wiley \& Sons, Nov. 1996. 
[31] T. M. Cover and J. A. Thomas, Elements of Information Theory, 1st ed. NY, USA: John Wiley \& Sons, 1991.

[32] J. Tellado, Multicarrier modulation with low PAR: applications to DSL and wireless. Norwell, MA, USA: Kluwer Academic Publishers, 2000.

[33] T. Chen, P. Catrysse, A. E. Gamal, and B. Wandell, "How small should pixel size be?" in Proceedings of the SPIE Electronic Imaging Conference, vol. 3965, San Jose, CA, USA, Jan. 2000.

[34] G. C. Holst, CCD Arrays, Cameras, and Displays, 2nd ed. Bellingham, WA, USA: SPIE Optical Engineering Press, 1998. 